\title{
Determination of local polarization properties of biological samples in the presence of diattenuation by use of Mueller optical coherence tomography
}

\author{
Miloš Todorović, Shuliang Jiao, and Lihong V. Wang \\ Optical Imaging Laboratory, Department of Biomedical Engineering, Texas A\&M University, College Station, Texas 77843-3120
} George Stoica

Department of Pathobiology, Texas A\&M University, College Station, Texas 77843-5547

Received May 16, 2004

\begin{abstract}
A unique feature of polarization-sensitive Mueller optical coherence tomography is that, by measuring Jones or Mueller matrices, it can reveal the complete polarization properties of biological samples, even in the presence of diattenuation. We map local polarization properties for the first time to our knowledge by using polar decomposition in combination with least-squares fitting to differentiate measured integrated Jones matrices with respect to depth. We also introduce the new concept of dual attenuation coefficients to characterize diattenuation per unit infinitesimal length in tissues. We experimentally verify the algorithm using measurements of a section of porcine tendon and the septum of a rat heart. (C) 2004 Optical Society of America OCIS codes: $120.2130,170.1650,170.3880,170.4500,260.1440,260.5430,290.7050$.
\end{abstract}

Polarization-sensitive optical coherence tomography (OCT) has established itself as a promising noninvasive imaging modality that offers both high-resolution and multiple-contrast mechanisms. Birefringence contrast describes the anisotropy of the phase velocity of light in a sample that is inherent in a variety of biological components, such as collagen, myelin, and elastic fibers. ${ }^{1-7}$ However, the potential of birefringence contrast for determining the depth-resolved local polarization properties and the structures of samples has not been fully exploited because of the integrating nature of phase retardation along the optical path. In this Letter we present a new differentiation algorithm based on the polar decomposition of Jones matrices and the subsequent least-squares regression analysis. The algorithm is capable of extracting the polarization parameters of small segments at all depths along the axial scans (A scans) in the sample, even in the presence of diattenuation, for the first time to our knowledge.

Jiao et $a l{ }^{3}$ showed that a scattering sample acts as a nondepolarizing medium because of the interference-based heterodyne detection scheme used in OCT. Therefore the polarization properties of biological samples can be described equally by either Jones or Mueller matrices. For this study we used a fiber-based polarization-sensitive Mueller OCT system (Fig. 1) to obtain Jones matrices. The lowcoherence light source is a horizontally polarized superluminescent diode $\left(\lambda_{0}=850 \mathrm{~nm}, \mathrm{FWHM}=26 \mathrm{~nm}\right.$, output power $5 \mathrm{~mW}$ ). The polarization of the beam is continuously modulated by a polarization modulator whose fast axis is set at $45^{\circ}$. The modulated light is split and delivered through single-mode fibers to the reference delay line and the sample. Polarization of the light emerging from the reference arm is controlled by a linear polarizer set at $45^{\circ}$. As the backreflected reference and sample beams interfere in the detection arm, the horizontal and vertical polarization components of the combined beam are detected by two photodiodes, respectively. The details of the experimental system and the algorithm for calculating the Jones matrices of a sample will be reported elsewhere. ${ }^{8}$

The Jones matrix for a given depth along each A scan obtained from Mueller OCT encompasses the integrated effect of all the segments within the depth:

$$
\mathbf{J}_{\mathrm{emi}}=\mathbf{J}_{\mathrm{st} 1}^{T} \ldots \mathbf{J}_{\mathrm{st}(i-1)}^{T} \mathbf{J}_{\mathrm{rti}} \mathbf{J}_{\mathrm{st}(i-1)} \ldots \mathbf{J}_{\mathrm{st} 1}
$$

where index emi denotes the expected measured Jones matrix based on the signals backscattered from the $i$ th segment, sti represents the one-way or actual Jones matrix of the $i$ th segment, and rti denotes the round-trip matrix of the $i$ th segment $\left(\mathbf{J}_{\mathrm{rti}}=\mathbf{J}_{\mathrm{sti}}^{T} \mathbf{J}_{\mathrm{sti}}\right)$. Algebraically, we can recover any round-trip single-segment matrix by recovering the one-way single-segment matrices of the preceding segments one by one and eliminating their integrating effects:

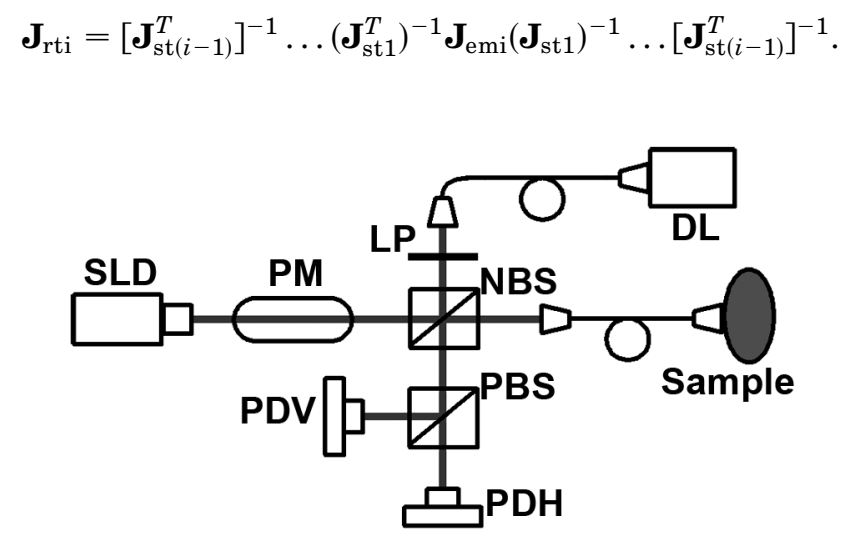

Fig. 1. Schematic of the Mueller OCT system. PM, polarization modulator; NBS, nonpolarizing beam splitter; PBS, polarizing beam splitter; LP, linear polarizer; DL, delay line; PDH, PDV, photodiodes for horizontal and vertical polarizations, respectively; SLD, superluminescent diode. 
We need to assume a model of a tissue segment to proceed with characterizing its properties. A sequence of a linear diattenuator and a linear retarder can model a thin segment of biological tissue well. This model is reasonable because physical properties, such as the orientation of the collagen fibers, are almost constant at the scale of OCT resolution $(\sim 10 \mu \mathrm{m})$. It is further assumed that the orientations of the retarder and diattenuator are the same, which is logical since their orientations are directly related to the orientation of the tissue fibers. In this case the order between the diattenuator and retarder is irrelevant, and the thin tissue segment is referred to as homogeneous in the sense of polarimetry. The equivalent one-way Jones matrix of a segment is given by

$\mathbf{J}_{\mathrm{st}}=\left[\begin{array}{cc}Q_{x} \cos ^{2} \theta+Q_{y} \sin ^{2} \theta & \left(Q_{x}-Q_{y}\right) \sin \theta \cos \theta \\ \left(Q_{x}-Q_{y}\right) \sin \theta \cos \theta & Q_{x} \sin ^{2} \theta+Q_{y} \cos ^{2} \theta\end{array}\right]$,

where $\varphi$ is the phase retardation; $\theta$ is the orientation angle of the fast eigenvector for both the diattenuator and the retarder; and $p_{x}$ and $p_{y}$ are the transmittances of the fast and slow eigenvectors, respectively. The round-trip single-segment Jones matrix closely resembles the one-way matrix except that the transmittances are $p_{x}^{\prime}=p_{x}^{2}$ and $p_{y}^{\prime}=p_{y}^{2}$, and the retardation is doubled to $\varphi^{\prime}=2 \varphi$. The orientation retains its original value $\theta$. Therefore it is not necessary to obtain the one-way matrix to calculate the values of $\varphi, \theta, p_{x}$, and $p_{y}$ because the round-trip matrix will suffice.

The polar decomposition theorem, when used with Jones matrices, states that any nondepolarizing polarization element described by a Jones matrix can be represented as a cascade of a diattenuator and a retarder. ${ }^{9}$ The polar decomposition yields two matrices - a unitary matrix $\mathbf{U}$ corresponding to a retarder and a nonnegative definite Hermitian matrix $\mathbf{H}$ corresponding to a diattenuator. After the round-trip matrices of the individual segments are calculated, we can apply the polar decomposition to obtain matrices $\mathbf{U}$ and $\mathbf{H}$. To calculate the parameters of an individual segment, we fit the matrices $\mathbf{U}$ and $\mathbf{H}$ to the model Jones matrices of a linear retarder and a linear diattenuator in the least-squares sense, respectively.

Knowing the Jones matrices for the retarder $\left(\varphi^{\prime}=2 \varphi, \theta\right)$ and diattenuator $\left(p_{x}^{\prime}=p_{x}^{2}, p_{y}^{\prime}=p_{y}^{2}, \theta\right)$ and having the decomposed measured round-trip matrix of the individual segment in the form $\mathbf{J}_{m}=\mathbf{U}_{m} \mathbf{H}_{m}$, one can apply the least-squares method that minimizes the difference between the measured and the expected Jones matrices to calculate the parameters of interest. Sums $S_{1}$ and $S_{2}$ shown below are the base equations of the least-squares algorithm for calculating the parameters of the equivalent retarder and diattenuator, respectively:

$$
\begin{aligned}
& S_{1}=\sum_{i=1, j=1}^{2,2}\left(U_{m i, j}-J_{\text {retarder } i, j}\right)^{2}, \\
& S_{2}=\sum_{i=1, j=1}^{2,2}\left(H_{m i, j}-J_{\text {diattenuator } i, j}\right)^{2} .
\end{aligned}
$$

Minimization of $S_{1}$ with respect to $\theta$ and $\varphi$ and $S_{2}$ with respect to $p_{x}$ and $p_{y}$ yields expressions for calculating the orientation, retardation, and transmission parameters as shown below:

$$
\begin{aligned}
\tan 2 \theta= & \frac{U_{m 12}^{i}+U_{m 21}^{i}}{U_{m 11}^{i}-U_{m 22}^{i}}, \\
\cot \varphi= & \frac{U_{m 11}^{r}+U_{m 22}^{r}}{\left(U_{m 11}^{i}-U_{m 22}^{i}\right) \cos 2 \theta+\left(U_{m 12}^{i}+U_{m 21}^{i}\right) \sin 2 \theta} \\
p_{x}= & {\left[H_{m 11}^{r} \cos ^{2} \theta+H_{m 22}^{r} \sin ^{2} \theta\right.} \\
& \left.+\left(H_{m 12}^{r}+H_{m 21}^{r}\right) \sin \theta \cos \theta\right]^{1 / 2} \\
p_{y}= & {\left[H_{m 11}^{r} \sin ^{2} \theta+H_{m 22}^{r} \cos ^{2} \theta\right.} \\
& \left.-\left(H_{m 12}^{r}+H_{m 21}^{r}\right) \sin \theta \cos \theta\right]^{1 / 2}
\end{aligned}
$$

Given amplitude transmittances $p_{x}$ and $p_{y}$, we can calculate the diattenuation for each segment as $D=$ $\left|p_{x}^{2}-p_{y}^{2}\right| /\left(p_{x}^{2}+p_{y}^{2}\right)$. Because $p_{x, y}$ and $D$ are integrated parameters over the finite path length, we define the dual attenuation coefficients $\mu_{a x}$ and $\mu_{a y}$ as fractions of the intensity attenuation per unit infinitesimal length along the fast and slow axes, respectively, each having a unit of inverse length and being independent of the finite path length. In the case of homogeneous properties along an A scan we can extend Beer's law to $p_{x, y}^{2}=\exp \left(-\mu_{a x, y} t\right)$, where $t$ is the thickness of the sample. We can also calculate diattenuation as $D=\tanh \left(\left|\mu_{a x}-\mu_{a y}\right| t / 2\right)$.

The differentiation algorithm was applied to calculate polarization parameters of biological samples including a section of homogeneous porcine tendon and a piece of heterogeneous septum from a rat heart. The Jones matrices were measured with the Mueller OCT system with the dynamic calibration technique for eliminating the effects of birefringence of the single-mode fiber. ${ }^{10}$ To convert from optical path length to physical depth, we assumed the index of refraction to be 1.4 .

Figure 2 shows the phase retardation images before the differentiation (Fig. 2a) and after the differentiation algorithm (Fig. 2b) was applied, as well as the profiles of the corresponding retardation (Fig. 2c) and slow axis orientation (Fig. 2d) averaged across all

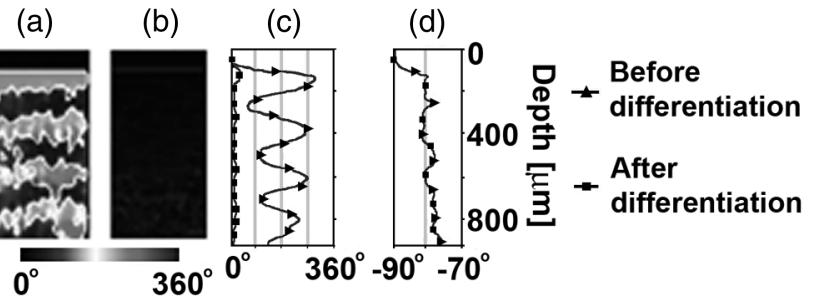

Fig. 2. Retardation images of porcine tendon (a) before differentiation and (b) after differentiation; (c) profiles of averaged phase retardation before and after differentiation; and (d) profiles of averaged slow-axis orientation before and after differentiation. Dimensions of each image are $0.5 \mathrm{~mm} \times 0.9 \mathrm{~mm}$ (width $\times$ height). 


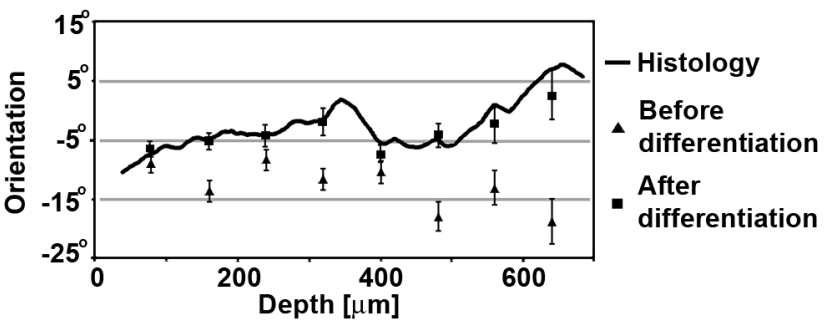

Fig. 3. Profiles of muscle-fiber orientation along one A scan obtained from histological images from Mueller OCT after differentiation and before differentiation.

50 A scans. The periodic structure in the retardation image in Fig. 2a, resulting from the $360^{\circ}$ phase wrapping, is a manifestation of the integrating nature of the phase retardation. Previously published images of porcine tendon showed $180^{\circ}$ phase wrapping patterns; inspection of the fast-axis orientation revealed an abrupt $90^{\circ}$ shift at each point of phase wrapping. This is consistent with the fact that a retarder $\mathbf{J}(\varphi=\pi+\delta, \theta)$ is equivalent to a retarder $\mathbf{J}(\varphi=\pi-\delta, \theta+\pi / 2)$; hence a phase wrapping of $180^{\circ}$ induces an orientation shift of $90^{\circ}$. The extension of the phase retardation range to $360^{\circ}$ in this study was achieved by keeping the depth profile of the orientation smooth. As a result, the differentiated retardation image shown in Fig. $2 b$ is nearly uniform. The differentiated phase retardation of $\sim 15^{\circ}$ per $10-\mu \mathrm{m}$ segment length (birefringence of $3.5 \times 10^{-3}$ ) is comparable with previously reported values ${ }^{2,5}$ and shows no periodic structures any more. The slow-axis orientation is nearly constant and corresponds to the orientation of the type I collagen fibers in tendons. This allowed us to calculate attenuation coefficients for porcine tendon (95\% confidence interval): $\mu_{a x}=$ $0.19 \pm 0.02 \mathrm{~mm}^{-1}, \mu_{a y}=0.29 \pm 0.02 \mathrm{~mm}^{-1}$, and the corresponding round-trip diattenuation $D$ is $0.10 \pm$ 0.02 for the 1 -mm-thick tendon sample. Note that the higher attenuation is along the collagen fibers (the slow axis).

Finally, we investigated the phase retardation and changing orientation of muscle fibers in the septum of a rat heart. The heart tissue was fixed in $10 \%$ buffered neutral formalin before imaging. Upon completion of the OCT imaging, the paraffin-embedded tissue was sectioned into $20-\mu \mathrm{m}$-thick layers and stained with hematoxylin and eosin. The stained sections were examined under an Olympus light microscope with $40 \times$ magnification to quantify the orientation of muscle fibers at various depths, where all the angles were measured with respect to the horizontal polarization axis. Figure 3 presents the results of the analysis of the septum from the surface up to a depth of $680 \mu \mathrm{m}$ along one A scan. To improve the signal-to-noise ratio (SNR), the A scan used to calculate the orientation and phase retardation is a result of averaging 50 measurements of the same A scan. Slow-axis orientation values obtained with the differentiation algorithm are in good agreement with the data from the histology. As expected, the standard deviation increases with depth because of the decreasing SNR. The muscle-fiber orientation values increase almost linearly from the surface up to $360 \mu \mathrm{m}$, where there is a negative change after which we again observe a linear increase. The discontinuity in the positive slope of the fiber orientation change may be the result of a trabeculata-compacta interface ${ }^{11}$ in the region of the septum proximal to the ventricular wall where the measurement was taken. The linear change of orientation observed in our study agrees with the results of a study ${ }^{11}$ in which the author observed that the fiber orientation linearly changes from the surface of the septum toward the deeper regions. To emphasize the importance of the depthwise differentiation, we show the orientation calculated without differentiation from the same data set. It is obvious that the orientation calculated in this way does not reflect the actual orientation because the values do not follow the profile obtained from the histology. Phase retardation in the sample is constant with depth, and its values fall almost perfectly in a straight line with an average value of $0.84 \pm 0.02^{\circ}$ per $10-\mu \mathrm{m}$ segment (95\% confidence interval) corresponding to a birefringence of $1.95 \pm 0.05 \times 10^{-4}$.

In summary, we have introduced a novel algorithm that uses polar decomposition and least-squares fitting to recover one-way Jones matrices of individual segments within scattering media. The algorithm was successfully applied to recovering the local polarization parameters of biological samples such as the orientation of the collagen and muscle fibers and the diattenuation of the tendon. Future work will include the implementation of a speckle-reduction algorithm that should aid the differentiation by increasing the SNR. This algorithm opens a new window of opportunity for OCT imaging applications.

We thank J. Criscione for his helpful discussion of this paper. This project was sponsored by the National Institutes of Health grant R01 CA092415. L. V. Wang's e-mail address is LWang@tamu.edu.

\section{References}

1. M. R. Hee, D. Huang, E. A. Swanson, and J. G. Fujimoto, J. Opt. Soc. Am. B 9, 903 (1992).

2. J. F. de Boer, T. E. Milner, M. J. C. van Gemert, and J. S. Nelson, Opt. Lett. 22, 934 (1997).

3. S. Jiao, G. Yao, and L. V. Wang, Appl. Opt. 39, 6318 (2000).

4. S. Jiao and L. V. Wang, Opt. Lett. 27, 101 (2002).

5. S. Jiao and L. V. Wang, J. Biomed. Opt. 7, 350 (2002).

6. M. C. Pierce, B. H. Park, B. Cense, and J. F. de Boer, Opt. Lett. 27, 1534 (2002).

7. H. Ren, Z. Ding, Y. Zhao, J. Miao, J. S. Nelson, and Z. Chen, Opt. Lett. 27, 1702 (2002).

8. S. Jiao, M. Todorović, G. Stoica, and L. V. Wang submitted a paper called "Fiber-based polarization-sensitive Mueller-matrix optical coherence with continuous source-polarization" to Appl. Opt.

9. J. J. Gil and E. Bernabeu, Optik 76, 67 (1987).

10. S. Jiao, W. Yu, G. Stoica, and L. V. Wang, Opt. Lett. 28, 1206 (2003).

11. D. D. Streeter, in Handbook of Physiology: The Cardiovascular System (American Physiological Society, Bethesda, Maryland, 1979), pp. 61-112. 